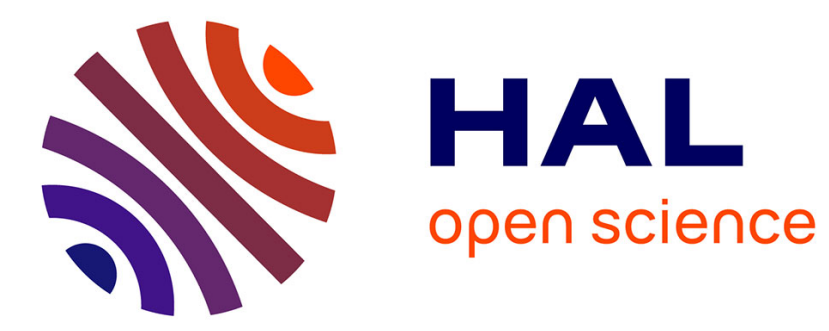

\title{
Uniqueness for multidimensional hyperbolic systems with commuting Jacobians
}

Hermano Frid, Philippe G. LeFloch

\section{To cite this version:}

Hermano Frid, Philippe G. LeFloch. Uniqueness for multidimensional hyperbolic systems with commuting Jacobians. 2006. hal-00121715

\section{HAL Id: hal-00121715 \\ https://hal.science/hal-00121715}

Preprint submitted on 21 Dec 2006

HAL is a multi-disciplinary open access archive for the deposit and dissemination of scientific research documents, whether they are published or not. The documents may come from teaching and research institutions in France or abroad, or from public or private research centers.
L'archive ouverte pluridisciplinaire HAL, est destinée au dépôt et à la diffusion de documents scientifiques de niveau recherche, publiés ou non, émanant des établissements d'enseignement et de recherche français ou étrangers, des laboratoires publics ou privés. 
To appear in : Archive for Rational Mechanics and Analysis.

\title{
UNIQUENESS FOR MULTIDIMENSIONAL HYPERBOLIC SYSTEMS WITH COMMUTING JACOBIANS
}

\author{
HERMANO FRID AND PHILIPPE G. LEFLOCH
}

\begin{abstract}
We consider nonlinear hyperbolic systems of conservation laws in several space dimensions whose Jacobian matrices commute and, more generally, systems that need not be conservative. Generalizing a theorem by Bressan and LeFloch for onedimensional systems, we establish that the Cauchy problem admits at most one entropy solution depending continuously upon its initial data. The uniqueness result is proven within the class (introduced here) of locally regular $B V$ functions with locally controled oscillation. These regularity conditions are modeled on well-known properties in the one-dimensional case. Our uniqueness theorem also improves upon the known results for one-dimensional systems.
\end{abstract}

\section{INTRODUCTION}

In this paper we consider hyperbolic systems of conservation laws

$$
\partial_{t} u+\sum_{k=1}^{d} \partial_{k} f^{k}(u)=0, \quad u=u(x, t) \in \mathcal{U}, \quad x \in \mathbb{R}^{d},
$$

where $\mathcal{U}$ is an open and bounded subset of $\mathbb{R}^{N}$, the flux-functions $f^{k}: \mathcal{U} \rightarrow \mathbb{R}^{N}$ are given smooth mappings, and the notation $x=$ $\left(x_{1}, \ldots, x_{d}\right), \partial_{k}:=\partial_{x_{k}}$ is used. We also treat their nonconservative generalization

$$
\partial_{t} u+\sum_{k=1}^{d} A^{k}(u) \partial_{k} u=0,
$$

where the matrices $A^{k}(u)$ need not be of the form $D f^{k}(u)$. When referring to (1.1) we also use the notation $A^{k}(u):=D f^{k}(u)$. Hyperbolicity for (1.1) and (1.2) means that, for every unit vector $\nu=\left(\nu_{k}\right) \in \mathbb{R}^{d}$ and for every state $u \in \mathcal{U}$, the matrix $\sum_{k=1}^{d} \nu_{k} A^{k}(u)$ admits $N$ real

2000 Mathematics Subject Classification. 35L65, 76L05.

Key words and phrases. conservation law, nonlinear hyperbolic system, entropy solution, uniqueness, continuous dependence, function with bounded variation, nonconservative product. 
(not necessarily distinct) eigenvalues $\lambda_{j}(u ; \nu)$ and a full basis of righteigenvectors $r_{j}(u ; \nu), 1 \leq j \leq N$. It is also assumed that the wave speeds are uniformly bounded in the sense that

$$
\sup _{\substack{1 \leq j \leq N \\ u \in \mathcal{U},|\nu|=1}}\left|\lambda_{j}(u ; \nu)\right|<\lambda^{\infty},
$$

where $|\nu|$ denotes the Euclidean norm of a vector $\nu$. (Of course, such a bound is always available on any compact subset of $\mathcal{U}$, at least.) Note that, for the results in this paper, the set $\mathcal{U}$ need not be "small" nor connected.

It is well-known that discontinuities appear in initially smooth solutions of (1.1) and that it is necessary to consider "entropy solutions" in the sense of distributions. Various entropy conditions have been introduced in the literature, depending on the properties of the coefficients $A^{k}$ of the system $[23,10,26,24]$. (We will be more specific in Sections 3 and 5 below.) Recall that, in one space dimension $(d=1)$ and when $\mathcal{U}$ is a small neighborhood of a constant state in $\mathbb{R}^{N}$ at least, Glimm's existence theory [18] indicates that the "natural" function space associated with (1.1) is the class BV of functions with bounded variation.

A general uniqueness theory is available in the case of one space dimension. Bressan and LeFloch [8] proved that the Cauchy problem for (1.1) (with $d=1$ ) admits at most one entropy solution satisfying the tame variation condition, which requires, in essence, that the total variation on an interval at a given time controls the total variation along any space-like curve included in the domain of determinacy of the given interval. The tame variation property is satisfied by solutions constructed, for instance, by the Glimm scheme ([27, 25, 20, 21, 28, 3] for recent works) or by the vanishing viscosity method [4], and, therefore, the theorem in [8] provides a uniqueness result in the same class where the existence is known. Later, it was observed $[7,9]$ that the uniqueness result remains true under even weaker conditions (tame oscillation or bounded variation on spacelike lines). These results assumed that all characteristic fields of (1.1) were genuinely nonlinear or linearly degenerate. The uniqueness theory was finally extended [2] to encompass hyperbolic systems with general flux-functions and (nonclassical) entropy solutions that do not satisfy standard entropy criteria, as well as to nonconservative systems of the form (1.2) (with $d=1$ ). See [24] for details.

By contrast, very little is known on systems in several space dimensions. It has been pointed out by Rauch [29] based on an earlier theorem by Brenner [5] that the Cauchy problem is never well-posed in $B V$ unless the matrices $A^{k}(u)$ commute. Dafermos recognized the 
importance of the class of hyperbolic systems with commuting Jacobians, as a first milestone toward developing a general theory for multidimensional hyperbolic systems (although this class does not seem to include examples of direct interest in continuum physics).

For these systems, Dafermos [12] was able to derive uniform a priori bounds on entropy solutions, especially $L^{p}$ bounds in the case $N=2$ and $d \geq 1$, for all $p \geq 1$. One key property of such systems is the existence of a common basis of eigenvectors for all matrices $A^{k}(u)$. It is conceivable that the existence of $B V$ solutions could be established for systems with commuting Jacobians, when initial data have small total variation. The recent work on multidimensional, rotationally invariant systems ([1] and the references therein) represents one very interesting step in this direction, although solutions of such systems may not always have bounded variation due to a loss of strictly hyperbolicity and other degeneracies.

The aim of the present paper is to generalize the one-dimensional uniqueness theory to multidimensional systems, under the structure assumption that the matrices $A^{k}(u)$ in (1.1) and (1.2) commute. We refer to this class as systems with "commuting Jacobians" although, in (1.2), the matrices $A^{k}(u)$ need not be Jacobian matrices. For reasons explained below, we consider solutions that are slightly better behaved than $B V$. Roughly speaking, we call a $B V$ function locally regular if it admits traces (at all but a set of zero measure for the $(d-1)$ dimensional Hausdorf measure $\mathcal{H}^{d-1}$ ) in the pointwise sense rather than in the averaged $L^{1}$ sense as is the case for arbitrary functions with bounded variation. In addition, following previous experience with one-dimensional systems, we assume that the solutions have locally controled oscillation in the sense that, loosely speaking, for almost every $t>0$, for each $x \in \mathbb{R}^{d}$, and for any $\varepsilon>0$, there exists a $r_{0}>0$ such that $\varepsilon$ plus the oscillation of $u(t)$ in any ball $B(x ; r)$ (with $0<$ $\left.r \leq r_{0}\right)$ controls the oscillation in the forward domain of dependence of $B(x ; r)$.

For one-dimensional systems, solutions to the Cauchy problem with initial data with small total variation satisfy the tame variation condition and, therefore, our (much weaker) locally controled oscillation condition. The regularity results established by Glimm and Lax [19], DiPerna [15], Dafermos [11, 13], and Liu [27] imply that the entropy solutions to systems of conservation laws are actually $B V$ locally regular with locally controled oscillation.

Our aim is proving that, within the class of locally regular $B V$ functions with locally controled oscillation (see precise definitions in Section 
2 below), the Cauchy problem associated with a system with commuting Jacobian (1.1)-(1.2) has at most one solution depending continuously on its initial data. Our method of proof is based on a suitable generalization of the arguments in [8] to the multidimensional setting. Part of the analysis consists of writing a suitable decomposition of a function of bounded variation $v=v(x), x \in \mathbb{R}^{d}$, which exhibits regions of large or small local oscillation for $v$. While this is elementary when $d=1$ (and the decomposition can actually be based on the total variation), this is no longer so when $d>1$ and this explains why we have to restrict attention in this paper to the class of locally regular $B V$ functions.

The regularity conditions are modeled on stronger properties satisfied in the one dimensional case, and it is conceivable that, in the multidimensional case and for systems with commuting Jacobians considered in this paper, these regularity assumptions may eventually be established together with the existence theory.

\section{Preliminary Definitions and notations}

Let $w \in B V\left(\mathbb{R}^{d}\right)$ (the space of integrable functions with bounded variation). It is well known that the function $w$ induces a decomposition of $\mathbb{R}^{d}$ of the form (e.g. Federer [17])

$$
\mathbb{R}^{d}=\mathcal{C}_{w} \cup \mathcal{J}_{w} \cup \mathcal{E}_{w},
$$

where $\mathcal{C}_{w}$ is the set of points of approximate continuity of $w, \mathcal{J}_{w}$ the set of approximate jump discontinuity, and $\mathcal{H}^{d-1}\left(\mathcal{E}_{w}\right)=0$. The set $\mathcal{J}_{w}$ is $\mathcal{H}^{d-1}$-rectifiable and so, except for a set of $\mathcal{H}^{d-1}$-measure zero, can be covered with a countable family, $\left\{\mathcal{S}_{w}^{\alpha}\right\}$, of graphs of functions of class $C^{1}, \gamma_{w}^{\alpha}: \mathbb{R}^{d-1} \rightarrow \mathbb{R}$, that is,

$$
\mathcal{J}_{w} \subset\left(\bigcup_{\alpha} \mathcal{S}_{w}^{\alpha}\right) \cup \mathcal{N}_{w}, \quad \mathcal{H}^{d-1}\left(\mathcal{N}_{w}\right)=0 .
$$

We then define the jump of $w$ on the graph $\mathcal{S}_{w}^{\alpha}$ (restricted to the ball $\left.B_{R}\right)$ as

$$
\left[\mathcal{S}_{w}^{\alpha}\right]_{R}:=\operatorname{ess} \sup \left\{|[w(x)]|: x \in \mathcal{S}_{w}^{\alpha} \cap B_{R}\right\},
$$

where the (essential) supremum is taken with respect to the measure $\mathcal{H}^{d-1}\left\lfloor\left(\mathcal{S}_{w}^{\alpha} \cap B_{R}\right)\right.$, and $B_{R}$ is the open ball in $\mathbb{R}^{d}$ of radius $R$ centered at the origin. As usual, $[w(x)]:=w_{+}(x)-w_{-}(x)$, where $w_{ \pm}(x)$ are the approximate limits from each side of the tangent hyperplane at $x$, determined according to a continuous, unit, normal vector field $\nu_{w}^{\alpha}(x)$ to $\mathcal{S}_{w}^{\alpha}$. 
Given an open set $\Omega \subset \mathbb{R}^{d}$ and a point $x \in \bar{\Omega}$ the local oscillation at the point $x$ (of the restriction of $w$ to the set $\bar{\Omega}$ ) is defined by

$$
\operatorname{osc}_{x}(w \mid \bar{\Omega}):=\limsup _{\rho \rightarrow 0} \operatorname{osc}(w \mid \bar{\Omega} \cap B(x ; \rho)),
$$

where, for any measurable set $A$, the oscillation of $w$ in the set $A$ is

$$
\operatorname{osc}(w \mid A):=\operatorname{ess} \sup _{x \in A} w(x)-\operatorname{ess} \inf _{x \in A} w(x) .
$$

The standard notions of approximate continuity points and approximate jump points of general BV functions are based on the (averaged) $L^{1}$ norm. We now introduce a class of BV functions which admit values at continuity points as well as left- and right-hand limits in a classical, pointwise sense.

Definition 2.1. We say that $w \in B V\left(\mathbb{R}^{d}\right)$ is a locally regular $B V$ function with respect to some countable family of graphs of $C^{1}$-functions, $\left\{\mathcal{S}_{w}^{\alpha}\right\}$, and we write $u \in B V^{\mathrm{loc}}\left(\mathbb{R}^{d}\right)$, if the graphs $\mathcal{S}_{w}^{\alpha}$ cover $\mathcal{J}_{w}$ in the sense (2.2) and the following property holds true. Given any $\varepsilon, R>0$, let $\left\{\mathcal{S}_{w, \varepsilon, R}^{\beta}\right\}$ denote the smallest subfamily of $\left\{\mathcal{S}_{w}^{\alpha} \cap B_{R}\right\}$ containing all sets $\mathcal{S}_{w}^{\alpha} \cap B_{R}$ where the jump of the function $w$ is greater than $\varepsilon$,

$$
\left[\mathcal{S}_{w}^{\alpha}\right]_{R}>\varepsilon
$$

and let $S_{w, \varepsilon, R}$ be the union of all sets in this subfamily. Then $\left\{\mathcal{S}_{w, \varepsilon, R}^{\beta}\right\}$ should be finite and, for some positive integer $N_{\varepsilon}$, there should be a decomposition

$$
B_{R} \backslash S_{w, \varepsilon, R}=: \bigcup_{i=1}^{N_{\varepsilon}} \Omega_{i}^{\varepsilon},
$$

where $\Omega_{i}^{\varepsilon}$ are open sets and $w$ has small local oscillation at each point $x \in \bar{\Omega}_{i}^{\varepsilon}, 1 \leq i \leq N_{\varepsilon}$,

$$
\operatorname{Osc}_{x}\left(w \mid \bar{\Omega}_{i}^{\varepsilon}\right) \leq \varepsilon
$$

Remark 2.1. Clearly, when $d=1$ we have $B V^{\text {loc }}(\mathbb{R})=B V(\mathbb{R})$.

Proposition 2.1. (Pointwise traces of locally regular BV functions.) Assume that $w \in B V^{\text {roc }}\left(\mathbb{R}^{d}\right)$, with respect to a countable family of graphs of $C^{1}$-functions $\left\{\mathcal{S}_{w}^{\alpha}\right\}$. Then, for all $x \in \mathbb{R}^{d}$ out of a set of $\mathcal{H}^{d-1}$ measure zero contained in $\bigcup_{\alpha} \mathcal{S}_{w}^{\alpha}$, either $w$ is continuous at $x$ or else $x \in \mathcal{S}_{w}^{\alpha_{0}}$ for some $\alpha_{0}$ and the following limits exist

$$
\lim _{\substack{y \rightarrow x \\ y \in\left(\mathcal{S}_{w}^{x_{0}}\right)_{ \pm}}} w(y)=w_{ \pm}(x)
$$

where $\left(\mathcal{S}_{w}^{\alpha}\right)_{+}$and $\left(\mathcal{S}_{w}^{\alpha}\right)_{-}$denote the two disjoint parts of $\mathbb{R}^{d}$ above and below the graph $\mathcal{S}_{w}^{\alpha}$. 
Proof. It suffices to prove the assertion for $x \in B_{R}$, with arbitrary $R$. First, if $x \in \mathcal{C}_{w} \cap B_{R}$ then, for any $n \in \mathbb{N}, x \notin S_{w, 1 / n, R}$ and, so, for each $n$ we may find $r_{n}>0$ such that $\operatorname{osc}\left(w \mid B\left(x ; r_{n}\right)\right) \leq 1 / n$ and, hence, $w$ is continuous at $x$.

Second, if $x \in \mathcal{J}_{w} \cap \bigcup_{\alpha} \mathcal{S}_{w}^{\alpha}$, then $x \in S_{w, 1 / n, R}$ for all $n$ greater than some fixed $n_{0}$ (determined by $|[w(x)]|>1 / n_{0}$ ). The sets $\Omega_{i}^{1 / n}$ introduced in (2.3) have piecewise $C^{1}$ boundary. It is not restrictive to suppose that $x$ belongs to the smooth part of the boundary of exactly two such open sets, for each $n$ greater than $n_{0}$ since this is true for all $x \in \mathcal{J}_{w} \cap \bigcup_{\alpha} \mathcal{S}_{w}^{\alpha}$, out of a set of $\mathcal{H}^{d-1}$-measure zero. For such a point $x$, there exists $\alpha_{0}$ such that $x \in \mathcal{S}_{w}^{\alpha_{0}}$ and for any $n$ sufficiently large there is $r_{n}>0$ such that

$$
\operatorname{osc}\left(u \mid B\left(x ; r_{n}\right) \cap\left(\mathcal{S}_{w}^{\alpha_{0}}\right)_{ \pm}\right) \leq \frac{1}{n},
$$

which proves the existence of the limits in (2.4) and concludes the proof.

Let $\mathcal{U} \subset \mathbb{R}^{N}$ be any bounded open set and $T>0$. We consider functions $u: \mathbb{R}^{d} \times[0, T] \rightarrow \mathcal{U}$ such that

$$
u \in L^{\infty}\left((0, T) ; B V\left(\mathbb{R}^{d}\right)\right) \cap \operatorname{Lip}\left([0, T] ; L^{1}\left(\mathbb{R}^{d}\right)\right) .
$$

From (2.5) it follows that $u \in B V\left(\mathbb{R}^{d} \times(0, T)\right)$ and $u$ induces a decomposition of $\mathbb{R}^{d} \times(0, T)$,

$$
\mathbb{R}^{d} \times(0, T)=\mathcal{C}_{u} \cup \mathcal{J}_{u} \cup \mathcal{E}_{u},
$$

where we use the notation $\mathcal{C}_{u}, \mathcal{J}_{u}, \mathcal{E}_{u}$ introduced earlier. We want to establish a correspondence between the decomposition associated with the function $u$ and the decompositions (of the type (2.1)) associated with the functions $u(t)$ for $t \in(0, T)$. This will lead us to Definition 2.4, below.

Remark 2.2. We remark that, from $(2.5), u(t) \in B V\left(\mathbb{R}^{d}\right)$ for all $t \in$ $[0, T]$ and the total variation of $u(t)$ with respect to the space variables is uniformly bounded in $[0, T]$. Also, we can extend the decomposition (2.6) to $\mathbb{R}^{d} \times[0, T]$ by extending in a natural way the notions of approximate continuity and approximate jump discontinuity to the boundary hyperplans $\mathbb{R}^{d} \times\{0\}$ and $\mathbb{R}^{d} \times\{T\}$. For instance, we say that $u$ is approximately continuous at $\left(x_{0}, 0\right)$, denoting $\left(x_{0}, 0\right) \in \mathcal{C}_{u}$, if

$$
\lim _{\rho \rightarrow 0} \frac{1}{\rho\left|B\left(x_{0} ; \rho\right)\right|} \int_{0}^{\rho} \int_{B\left(x_{0} ; \rho\right)}\left|u(x, t)-u\left(x_{0}, 0\right)\right| d x d t=0,
$$

and $\left(x_{0}, 0\right)$ is said to be a point of approximate jump discontinuity, or, in short, $\left(x_{0}, 0\right) \in \mathcal{J}_{u}$, if for some unity vector $\bar{\nu} \in \mathbb{R}^{d+1}$ and two vectors 
$u_{+}, u_{-} \in \mathcal{U}$ we have

$$
\lim _{\rho \rightarrow 0} \frac{1}{\rho\left|B\left(x_{0} ; \rho\right)\right|} \int_{0}^{\rho} \int_{B\left(x_{0} ; \rho\right)}\left|u(x, t)-\bar{u}_{\bar{\nu}}\left(x-x_{0}, t\right)\right| d x d t=0
$$

where

$$
\bar{u}_{\bar{\nu}}(x, t)= \begin{cases}u_{-}, & (x, t) \cdot \bar{\nu}<0 \\ u_{+}, & (x, t) \cdot \bar{\nu}>0\end{cases}
$$

Analogously, we define the same notions for points in the boundary hyperplane $\mathbb{R}^{d} \times\{T\}$.

We assume that:

(A1) (Spacelike property.) There is a countable family $\left\{\mathcal{S}_{u}^{\alpha}\right\}$ of graphs of $C^{1}$-functions $\gamma_{u}^{\alpha}: \mathbb{R}^{d-1} \times \mathbb{R} \rightarrow \mathbb{R},(\bar{y}, t) \mapsto \gamma_{u}^{\alpha}(\bar{y}, t)$, in which the variable $t$ is one of the parameters, such that

$$
\mathcal{J}_{u} \subset\left(\bigcup_{\alpha} \mathcal{S}_{u}^{\alpha}\right) \cup \mathcal{N}_{u}, \quad \mathcal{H}^{d}\left(\mathcal{N}_{u}\right)=0 .
$$

Moreover, there is $\lambda>0$ such that

$$
\left|\gamma_{u}^{\alpha}\left(\bar{y}, t_{1}\right)-\gamma_{u}^{\alpha}\left(\bar{y}, t_{2}\right)\right|<\lambda\left|t_{1}-t_{2}\right|, \quad \forall \bar{y} \in \mathbb{R}^{d-1}, t_{1}, t_{2} \in[0, T] .
$$

(A2) (BV locally regular property at fixed time.) For almost every $t \in[0, T)$, the function $u(t)$ belongs to $B V^{\text {reg }}\left(\mathbb{R}^{d}\right)$ with respect to $\left\{\mathcal{S}_{u(t)}^{\alpha}\right\}$, where $\mathcal{S}_{u(t)}^{\alpha}$ denotes the graph of the $C^{1}$-function $\gamma_{u(t)}^{\alpha}(y):=\gamma_{u}^{\alpha}(y, t)$. In particular, this implies that, for almost every $t \in[0, T)$,

$$
\mathcal{J}_{u(t)} \subset\left(\bigcup_{\alpha} \mathcal{S}_{u(t)}^{\alpha}\right) \cup \mathcal{N}_{u(t)}, \quad \mathcal{H}^{d-1}\left(\mathcal{N}_{u(t)}\right)=0 .
$$

(A3) (Regular jump points.) For fixed $\alpha$, let $\mathcal{S}_{u}^{\alpha}$ be given by the parametrization

$$
y_{d}=\gamma_{u}(\bar{y}, t), \text { where } \bar{y}=\left(y_{1}, \ldots, y_{d-1}\right) .
$$

Let $t_{0} \in[0, T)$ be out of the exceptional null set in (A2), and let $\bar{y}_{0} \in \mathbb{R}^{d-1}$ be such that

$$
\left(\bar{y}_{0}, \gamma_{u\left(t_{0}\right)}\left(\bar{y}_{0}\right), t_{0}\right) \in \mathcal{J}_{u} \text { and }\left(\bar{y}_{0}, \gamma_{u\left(t_{0}\right)}\left(\bar{y}_{0}\right)\right) \in \mathcal{J}_{u\left(t_{0}\right)} \text {. }
$$

Then, for any $\varepsilon>0$ we should have some $\delta_{\varepsilon}>0$ with the following property. For $0<h_{0}<T-t_{0}$, define the function $\bar{u}(y, t)$, in the region $\left|\bar{y}-\bar{y}_{0}\right|<\delta_{\varepsilon},\left|y_{d}-\gamma_{u\left(t_{0}\right)}(\bar{y})\right| \leq \lambda h_{0}$ and $t_{0} \leq t \leq t_{0}+h_{0}$, by

$$
\bar{u}(y, t)= \begin{cases}u_{-}\left(\bar{y}, \gamma_{u\left(t_{0}\right)}(\bar{y})\right), & \text { if } \gamma_{u\left(t_{0}\right)}(\bar{y})-\lambda h_{0} \leq y_{d}<\gamma_{u}(\bar{y}, t), t_{0} \leq t \leq t_{0}+h_{0}, \\ u_{+}\left(\bar{y}, \gamma_{u\left(t_{0}\right)}(\bar{y})\right), & \text { if } \gamma_{u\left(t_{0}\right)}(\bar{y})+\lambda h_{0} \geq y_{d}>\gamma_{u}(\bar{y}, t), t_{0} \leq t \leq t_{0}+h_{0} .\end{cases}
$$


Then

$$
\begin{aligned}
\limsup _{h \rightarrow 0} \frac{1}{2 \lambda h^{2}} \int_{t_{0}}^{t_{0}+h} d t & \int_{-\lambda h}^{\lambda h} d s \int_{\substack{y_{d}-\gamma_{u\left(t_{0}\right)}(\bar{y})=s \\
\left|\bar{y}-\bar{y}_{0}\right|<\delta}}|u(y, t)-\bar{u}(y, t)| d \mathcal{H}^{d-1}(y) \\
& <\varepsilon \mathcal{H}^{d-1}\left(\mathcal{S}_{u\left(t_{0}\right), \bar{y}_{0}, \delta}^{\alpha}\right), \quad \text { for all } 0<\delta \leq \delta_{\varepsilon} \\
\text { where } \mathcal{S}_{u\left(t_{0}\right), \bar{y}_{0}, \delta}^{\alpha}: & =\mathcal{S}_{u\left(t_{0}\right)}^{\alpha} \cap\left\{y \in \mathbb{R}^{d}:\left|\bar{y}-\bar{y}_{0}\right|<\delta\right\} .
\end{aligned}
$$

Remark 2.3. Assumption (A1) requires that the $C^{1}$-functions whose

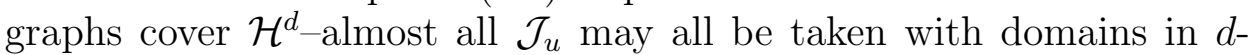
dimensional subspaces of $\mathbb{R}^{d+1}$ containing the $t$-axis (that is, the graphs are spacelike). In the forthcoming discussion, $u$ will denote a $B V$ solution of a hyperbolic system ((1.1) or (1.2)), for which the principle of finite speed of propagation applies. Therefore, Assumption (A1) is natural and follows from the above principle. In fact, at points $(x, t) \in \mathcal{J}_{u}$ where a tangent space to $\mathcal{J}_{u}$ is defined, with unit normal $\nu(x, t)$, we must have $\left|\nu(x, t) \cdot \mathbf{e}_{d+1}\right|<\delta_{\infty}<1$, with $\delta_{\infty}$ independent of $(x, t)$, where $\mathbf{e}_{d+1}$ is the (forward) unit vector in the direction of the $t$-axis.

Remark 2.4. Assumption (A3), in the context referred to in the above remark, is coherent also with Assumption (A1) since, the (initial) values $u\left(t_{0}\right)_{ \pm}\left(\bar{y}, \gamma_{u\left(t_{0}\right)}(\bar{y})\right)$ being close to $u\left(t_{0}\right)_{ \pm}\left(\bar{y}_{0}, \gamma_{u\left(t_{0}\right)}\left(\bar{y}_{0}\right)\right)$, respectively, and the unit normal vectors to $T_{\left(\bar{y}, \gamma_{u\left(t_{0}\right)}(\bar{y})\right)}\left(\mathcal{S}_{u\left(t_{0}\right)}^{\alpha}\right)$ being also close, for $\mid \bar{y}-$ $\bar{y}_{0} \mid<\delta$, it is to be expected that the solution $u(y, t)$ behaves as in the one-dimensional case in the whole region $\left|y_{d}-\gamma_{u\left(t_{0}\right)}(\bar{y})\right| \leq \lambda h$ and $t_{0} \leq t \leq t_{0}+h$, for $h>0$ sufficiently small at least and with $\lambda=$ $\lambda^{\infty}$ (introduced in (1.3)). We also observe that Assumption (A3) is automatically true in the one-dimensional case since, in this case,

$$
\lim _{h \rightarrow 0} \frac{1}{h^{2}} \int_{t_{0}}^{t_{0}+h} \int_{y_{0}-\lambda h}^{y_{0}+\lambda h}|\bar{u}(y, s)-\overline{\bar{u}}(y, s)| d y d t=0,
$$

where

$$
\overline{\bar{u}}(y, t)= \begin{cases}u_{-}\left(y_{0}, t_{0}\right), & \text { if }(y, t) \in\left(T_{\left(y_{0}, t_{0}\right)}\left(\mathcal{S}_{u}^{\alpha}\right)\right)_{-}, \\ u_{+}\left(y_{0}, t_{0}\right), & \text { if }(y, t) \in\left(T_{\left(y_{0}, t_{0}\right)}\left(\mathcal{S}_{u}^{\alpha}\right)\right)_{+},\end{cases}
$$

due to the $C^{1}$-regularity of $\mathcal{S}_{u}^{\alpha}$, where $\left(T_{\left(y_{0}, t_{0}\right)}\left(\mathcal{S}_{u}^{\alpha}\right)\right)_{ \pm}$denote the halfspaces to the left and right of the tangent line $T_{\left(y_{0}, t_{0}\right)}\left(\mathcal{S}_{u}^{\alpha}\right)$.

Some technical lemmas are in order, before we can introduce our main definitions. For $u \in B V\left(\mathbb{R}^{d} \times(0, T)\right)$ and $0<s<T$, denote by $\mathcal{E}_{u}^{s}$ the points of the exceptional set $\mathcal{E}_{u}$ belonging to the hyperplane $\{t=s\}$ of $\mathbb{R}^{d} \times(0, T)$. 
Lemma 2.1. For $u \in B V\left(\mathbb{R}^{d} \times(0, T)\right)$ one has $\mathcal{H}^{d-1}\left(\mathcal{E}_{u}^{t}\right)=0$ for almost every $t \in(0, T)$.

Proof. This is a direct consequence of the fact that $\mathcal{H}^{d}\left(\mathcal{E}_{u}\right)=0$ and the inequality

$$
\int_{(0, T)}^{*} \mathcal{H}^{d-1}\left\{x:(x, t) \in \mathcal{E}_{u}\right\} d t \leq 2 \frac{\alpha(d-1)}{\alpha(d)} \mathcal{H}^{d}\left(\mathcal{E}_{u}\right),
$$

where $\alpha(m)$ is the $m$-dimensional Lebesgue measure of the $m$-dimensional unit ball and the superscript * indicates upper-integral; this inequality follows from Proposition 2.10.27 in [17].

Lemma 2.2. Let $u$ be a function satisfying (2.5), (A1), and (A2), and let $\mathcal{J}_{u}$ be the jump set of $u$ and $\mathcal{J}_{u(t)}$ be the jump set of $u(t), t \in(0, T)$. For $s \in(0, T)$, set $\mathcal{J}_{u}^{s}:=\mathcal{J}_{u} \cap\{t=s\}$. Then, for almost every $t \in(0, T)$ we have

$$
\mathcal{H}^{d-1}\left(\mathcal{J}_{u(t)} \backslash \mathcal{J}_{u}^{t}\right)=0
$$

Proof. By (A1) we have $\mathcal{J}_{u}^{t} \subset \bigcup_{\alpha} \mathcal{S}_{u(t)}^{\alpha} \cup \mathcal{N}_{u}^{t}$, where we have set $\mathcal{N}_{u}^{s}:=$ $\mathcal{N}_{u} \cap\{t=s\}$ and $\mathcal{N}_{u}$ is the same as in (A1). Since, by (2.10), with $\mathcal{E}_{u}$ replaced by $\mathcal{N}_{u}, \mathcal{H}^{d-1}\left(\mathcal{N}_{u}^{t}\right)=0$, for almost every $t \in(0, T)$, the result then follows from (2.8).

Definition 2.2. Let $u \in L^{\infty}\left((0, T) ; B V\left(\mathbb{R}^{d}\right)\right) \cap \operatorname{Lip}\left([0, T] ; L^{1}\left(\mathbb{R}^{d}\right)\right)$, satisfying (A1), (A2) and (A3) above. We say that $t \in(0, T)$ is not a time of interaction if $t$ is a time for which (A2) holds and $\mathcal{H}^{d-1}\left(\mathcal{E}_{u}^{t}\right)=\mathcal{H}^{d-1}\left(\mathcal{J}_{u(t)} \backslash \mathcal{J}_{u}^{t}\right)=0$; we denote the subset of $[0, T)$ consisting of such values of $t$ by $\mathcal{I}^{c}(u)$, and set $\mathcal{I}(u)=[0, T) \backslash \mathcal{I}^{c}(u)$. Here, we use the convention $\mathcal{E}_{u}^{0}:=\mathbb{R}^{d} \backslash\left(\mathcal{J}_{u}^{0} \cup \mathcal{C}_{u}^{0}\right)$, where $\mathcal{J}_{u}^{0}$ and $\mathcal{C}_{u}^{0}$ are defined according Remark 2.2 .

Remark 2.5. We observe that, in view of Lemmas 2.1 and 2.2, one has $\mathcal{H}^{1}(\mathcal{I}(u))=0$.

We now introduce a new condition which is concerned with the timedependence of functions.

We will use the following notation. For $\left(x_{0}, t_{0}\right) \in \mathbb{R}^{d} \times(0, T), r, \lambda>0$, the domain of determinacy of the point $\left(x_{0}, t_{0}\right)$ is

$$
D\left(x_{0}, t_{0}, r, \lambda\right):=\left\{(x, t) \in \mathbb{R}^{d} \times(0, T):\left|x-x_{0}\right|<r-\lambda\left(t-t_{0}\right), t \geq t_{0}\right\}
$$


and, for every open set $\Omega \subset \mathbb{R}^{d}$,

$$
\begin{array}{r}
D\left(\Omega, t_{0}, \lambda\right):=\left\{(x, t) \in \mathbb{R}^{d} \times(0, T): \text { there exists } x_{0} \in \Omega, r>0\right. \text { such that } \\
\left.B\left(x_{0}, r\right) \subset \Omega \text { and }(x, t) \in D\left(x_{0}, t_{0}, r, \lambda\right)\right\}, \quad(2.12)
\end{array}
$$

and, for $0 \leq t_{0}<s<T$,

$$
\Omega\left(s, t_{0}, \lambda\right)=\left\{(x, t) \in D\left(\Omega, t_{0}, \lambda\right): t=s\right\} .
$$

We will frequently omit $\lambda$ in the above notations whenever there is no possible confusion.

Definition 2.3. Let $u \in L^{\infty}\left((0, T) ; B V\left(\mathbb{R}^{d}\right)\right) \cap \operatorname{Lip}\left([0, T] ; L^{1}\left(\mathbb{R}^{d}\right)\right)$. We say that $u$ has locally controled oscillation in time if there exists $\lambda>0$ and a constant $C_{0}>0$ such that for all $t_{0} \in[0, T)$ we have that, for all open set $\Omega \subset \mathbb{R}^{d}$, with piecewise smooth boundary, and for all $x_{0} \in \bar{\Omega}$ and $\varepsilon>0$, there exists $r_{0}>0$ such that

$$
\operatorname{osc}\left(u \mid D\left(B\left(x_{0} ; r\right) \cap \Omega, t_{0}, \lambda\right)\right) \leq C_{0} \operatorname{osc}\left(u\left(t_{0}\right) \mid B\left(x_{0} ; r\right) \cap \Omega\right)+\varepsilon
$$

for all $0<r \leq r_{0}$.

Remark 2.6. It is straightforward to see that in the one-dimensional case, the notion of locally controled oscillation is weaker than that of tame oscillation adopted in [7], due to the presence of the parameter $\varepsilon$ in Definition 2.3.

Remark 2.7. Let $u \in L^{\infty}\left((0, T) ; B V\left(\mathbb{R}^{d}\right)\right) \cap \operatorname{Lip}\left([0, T] ; L^{1}\left(\mathbb{R}^{d}\right)\right)$ and satisfy (A1) and (A2). For fixed $\alpha$, let $\mathcal{S}_{u}^{\alpha}$ be given by the parametrization

$$
y_{d}=\gamma_{u}(\bar{y}, t), \text { where } \bar{y}=\left(y_{1}, \ldots, y_{d-1}\right) .
$$

Let $t_{0} \in(0, T)$ be out of the exceptional null set in (A2), and let $\bar{y}_{0} \in \mathbb{R}^{d-1}$ be such that the pointwise limits $u\left(t_{0}\right)_{ \pm}\left(\gamma_{u\left(t_{0}\right)}\left(\bar{y}_{0}\right)\right)$ exist and coincide, so that $u\left(t_{0}\right)$ is continuous at $\gamma_{u\left(t_{0}\right)}\left(\bar{y}_{0}\right)$. It is straightforward to see that if $u$ has locally controled oscillation in time then (2.9) holds for such $\left(\bar{y}_{0}, t_{0}\right)$. Indeed, it suffices to take $\delta_{\varepsilon}$ small enough so that the oscillation of $u\left(t_{0}\right)$ in the region $\left|\bar{y}-\bar{y}_{0}\right|<\delta_{\varepsilon}, \gamma_{u\left(t_{0}\right)}(\bar{y})-h<y_{d}<$ $\gamma_{u\left(t_{0}\right)}(\bar{y})+h$, is less than, say, $\varepsilon / 2$ if $h$ is small enough. Then, the locally controlled oscillation property will implies that the oscillation of $u$ in the region $t_{0} \leq t \leq t_{0}+h,\left|\bar{y}-\bar{y}_{0}\right|<\delta_{\varepsilon}, \gamma_{u\left(t_{0}\right)}(\bar{y})-h<y_{d}<$ $\gamma_{u\left(t_{0}\right)}(\bar{y})+h$, is less than $\varepsilon$ for $h$ sufficiently small, and so (2.9) follows. Also, the $\bar{y}_{0}$ satisfying either the conditions in assumption (A3) or else the conditions in this remark encompass $\mathcal{H}^{d-1}$-almost all of $\mathbb{R}^{d-1}$.

We now ready to introduce the class of functions which will be of interest for the main uniqueness result in this paper. 
Definition 2.4. We denote by $\mathcal{O}\left(0, T ; B V^{\text {loc }}\left(\mathbb{R}^{d}\right)\right)$ the class of all functions $u \in L^{\infty}\left(\mathbb{R}^{d} \times(0, T), \mathcal{U}\right)$ satisfying $(2.5)$, (A1), (A2), (A3) and possessing locally controled oscillation in time.

Remark 2.8. In the one-dimensional case $(d=1)$ and by the results in Glimm and Lax [19] and DiPerna [15], solutions of hyperbolic systems (1.1) obtained by Glimm's method (for instance) belong precisely to the class $\mathcal{O}\left(0, T ; B V^{\text {reg }}\left(\mathbb{R}^{d}\right)\right)$. In fact, the regularity properties in the one-dimensional case are even stronger. See $[19,15,13,27]$ for details.

\section{Hyperbolic SySTEMS OF CONSERVATION LAWS}

In this section we state the main uniqueness result of this paper in the case of conservative and genuinely nonlinear systems, i.e., the Cauchy problem admits at most one solution depending $L^{1}$ continuously upon its initial data, among all possible entropy solutions in the class $\mathcal{O}\left(0, T ; B V^{\text {rec }}\left(\mathbb{R}^{d}\right)\right)$. We begin by recalling some basic terminology. We say that a Lipschitz function $P: \mathcal{U} \rightarrow \mathbb{R}$ is an entropy for (1.1) with associated entropy flux $Q: \mathcal{U} \rightarrow \mathbb{R}^{d}$ if

$$
\nabla P(u) \nabla f^{k}(u)=\nabla Q^{k}(u) \quad \text { for } k=1, \cdots, d \text { and almost every } u \in \mathcal{U} \text {. }
$$

If $P$ is strictly convex we say that the pair $(P, Q)$ is a strictly convex entropy pair.

Definition 3.1. Consider a hyperbolic system of conservation laws (1.1) endowed with a strictly convex entropy pair $(P, Q)$. A function $u \in L^{\infty}\left(\mathbb{R}^{d} \times(0, T) ; \mathcal{U}\right)$ is called an entropy solution of (1.1) if the equations (1.1) as well as the entropy inequality

$$
\partial_{t} P(u)+\sum_{k=1}^{d} \partial_{k} Q^{k}(u) \leq 0
$$

hold in the sense of distributions in $\mathbb{R}^{d} \times(0, T)$.

We consider entropy solutions of $(1.1)$ in $\mathcal{O}\left(0, T ; B V^{\text {loc }}\left(\mathbb{R}^{d}\right)\right)$ with a prescribed initial data

$$
\left.u(x, t)\right|_{t=0}=u_{0}(x),
$$

where $u_{0} \in B V\left(\mathbb{R}^{d} ; \mathcal{U}\right)$, which is assumed in the usual sense for functions in $\operatorname{Lip}\left([0, T] ; L^{1}\left(\mathbb{R}^{d}\right)\right)$. In what follows the parameter $\lambda$ appearing in (A3) and in (2.14), in the definition of the class $\mathcal{O}\left(0, T ; B V^{\text {loc }}\left(\mathbb{R}^{d}\right)\right)$, will always be assumed to be $\lambda^{\infty}$, that is, the corresponding function 
will belong to $\mathcal{O}\left(0, T ; B V^{\mathrm{loc}}\left(\mathbb{R}^{d}\right)\right)$ for $\lambda=\lambda^{\infty}$. As in [8] our uniqueness result is conditioned to the existence of a semigroup of entropy solutions, defined naturally as follows.

Definition 3.2. A continuous semigroup of entropy solutions of (1.1) is a mapping $S: \mathcal{K} \times[0, \infty) \rightarrow \mathcal{K}$ defined on a non-empty subset $\mathcal{K}$ of $B V\left(\mathbb{R}^{d} ; \mathcal{U}\right)$ such that the following three properties hold:

(1) (Semigroup property.) For all $t_{1}, t_{2} \geq 0$ and $u_{0} \in \mathcal{K}$, we have $S(0) u_{0}=u_{0}, S\left(t_{1}\right) u_{0} \in \mathcal{K}$ and $S\left(t_{2}\right) \circ S\left(t_{1}\right) u_{0}=S\left(t_{2}+t_{1}\right) u_{0}$.

(2) (Continuous dependence.) For some fixed constant $K>0$ and for all $u_{0}, v_{0} \in \mathcal{K}$ and $t_{1}, t_{2} \geq 0$,

$\left\|S\left(t_{2}\right) u_{0}-S\left(t_{1}\right) v_{0}\right\|_{L^{1}\left(\mathbb{R}^{d}\right)} \leq K\left(\left\|u_{0}-v_{0}\right\|_{L^{1}\left(\mathbb{R}^{d}\right)}+\left|t_{2}-t_{1}\right|\right)$.

(3) (Entropy solution.) For each function $u_{0} \in \mathcal{K}$ the function

$$
u^{*}(t):=S(t) u_{0}
$$

is an entropy solution of (1.1) belonging to the class

$$
\mathcal{O}\left(0, T ; B V^{\text {roc }}\left(\mathbb{R}^{d}\right)\right) .
$$

(4) (Consistency with single jump.) Let $\nu \in \mathbb{R}^{d}$, with $|\nu|=1$, and $\bar{u}_{0}$ be given by

$$
\bar{u}_{0}(x)= \begin{cases}u_{-}, & x \cdot \nu<0 \\ u_{+}, & x \cdot \nu>0\end{cases}
$$

and suppose there is $\sigma \in \mathbb{R}$ such that

$$
-\sigma\left(u_{+}-u_{-}\right)+\sum_{k=1}^{d} \nu_{k}\left(f^{k}\left(u_{+}\right)-f^{k}\left(u_{-}\right)\right)=0,
$$

and

$$
-\sigma\left(P\left(u_{+}\right)-P\left(u_{-}\right)\right)+\sum_{k=1}^{d} \nu_{k}\left(Q^{k}\left(u_{+}\right)-Q^{k}\left(u_{-}\right)\right) \leq 0 .
$$

Let $\bar{\nu}=\frac{1}{\sqrt{1+\sigma^{2}}}\left(\nu_{1}, \ldots, \nu_{d},-\sigma\right)$ and

$$
\bar{u}(x, t)=\left\{\begin{array}{ll}
u_{-}, & (x, t) \cdot \bar{\nu}<0 \\
u_{+}, & (x, t) \cdot \bar{\nu}>0
\end{array}, \quad(x, t) \in \mathbb{R}^{d} \times \mathbb{R}_{+} .\right.
$$

Then, we must have $\left[S(t)\left(\bar{u}_{0}\right)\right](x)=\bar{u}(x, t)$ for all $t>0, x \in \mathbb{R}^{d}$.

(5) (Consistency with regularity of the initial data) If, for $u_{0} \in \mathcal{K} \cap$ $B V^{\text {loc }}$ reg $\left(\mathbb{R}^{d}\right), u^{*}(x, t)=\left[S(t)\left(u_{0}\right)\right](x)$ and we have $\mathcal{H}^{d-1}\left(\mathcal{E}_{u^{*}}^{0}\right)=$ $\mathcal{H}^{d-1}\left(\mathcal{J}_{u_{0}} \backslash \mathcal{J}_{u^{*}}^{0}\right)=0$ then 0 is not a time of interaction for $u^{*}$, that is, 0 is not in the exceptional null set of (A2). 
(6) (Finite speed of propagation.) Given any open set $\Omega \subset \mathbb{R}^{d}$, the values of $u^{*}$ on $D\left(\Omega, t_{0}, \lambda^{\infty}\right)$ depend only on the values of $u^{*}\left(t_{0}\right)$ on $\Omega$, for all $t_{0} \geq 0$.

All of the properties above are standard for one-dimensional systems, and the existence of a continuous semi-group in that case is now wellknown. (See $[6,24]$ for references.) We expect that the same existence result could be obtained for multidimensional systems with commuting Jacobians, and it is the subject of this paper to establish the uniqueness of such solutions.

We now state our main uniqueness result for hyperbolic systems of conservation laws (1.1).

Theorem 3.1. (Main uniqueness result.) Assume that the system of conservation laws (1.1) admits a strictly convex entropy pair, has genuinely nonlinear characteristic fields (in the sense that

$$
\nabla \lambda_{j}(u ; \nu) \cdot r_{j}(u ; \nu) \neq 0
$$

for all $u \in \mathcal{U}$ and $j=1, \ldots, N)$, and has commuting Jacobian matrices. Suppose also that there exists a continuous semigroup of entropy solutions of $(1.1)$, defined on some non-empty subset $\mathcal{K}$ of $B V\left(\mathbb{R}^{d} ; \mathcal{U}\right)$. Then, given any $u_{0} \in \mathcal{K}$,

$$
u^{*}(t):=S(t) u_{0}
$$

is the only entropy solution $u$ of (1.1),(3.3) satisfying

$$
u \in \mathcal{O}\left(0, T ; B V^{\text {roc }}\left(\mathbb{R}^{d}\right)\right)
$$

and $u(t) \in \mathcal{K}$ for all $t \in[0, T]$ and $T>0$.

Theorem 3.1 improves and extends to the multidimensional context the main theorems in $[8,7]$. We prove it in the next section by following the general strategy adopted in [8]. The situation in several space dimensions is much more involved, since standard pointwise properties of functions in $B V(\mathbb{R})$ are not shared by arbitrary functions in $B V\left(\mathbb{R}^{d}\right)$ when $d>1$, and this precisely motivated the introduction of the class $B V^{\text {roc }}\left(\mathbb{R}^{d}\right)$. This, seemingly unavoidable, restriction on the class $B V\left(\mathbb{R}^{d}\right)$, by itself, does not make straightforward the generalization of the one-dimensional arguments: new ideas have to be developed to overcome several difficulties not present in the one-dimensional case, as we will see in Section 4.

We point out that the assumption of genuine nonlinearity is required if one expects a single entropy inequality to guarantee uniqueness for the Cauchy problem. For non-genuinely nonlinear systems, even in 
one space dimension, a single entropy inequality can not prevent the possibility of undercompressive, nonclassical shocks [24] and therefore multiple solutions to the Cauchy problem. In Section 5 below we discuss a framework which is adapted to this degree of generality.

\section{TANGENCY PROPERTY AND PROOF OF THE MAIN THEOREM}

The purpose of this section is to give the proof of Theorem 3.1. The decisive step is provided by the following theorem which is of independent interest (as noted in [2] when $d=1$ ).

Theorem 4.1. (Tangency property.) Consider the hyperbolic system (1.1) and assume that it has commuting Jacobian matrices, genuinely nonlinear characteristic fields, and is endowed with a strictly convex entropy pair. Let $u$ and $v$ be two entropy solutions in $\mathcal{O}\left(0, T ; B V^{\text {roc }}\left(\mathbb{R}^{d}\right)\right)$. If, for some $t_{0} \notin \mathcal{I}(u) \cup \mathcal{I}(v)$, one has

$$
u\left(t_{0}\right)=v\left(t_{0}\right) \text { almost everywhere in } \mathbb{R}^{d},
$$

then, for any $R>0$,

$$
\lim _{\substack{t \rightarrow t_{0} \\ t>t_{0}}} \frac{1}{t-t_{0}}\|u(t)-v(t)\|_{L^{1}\left(B_{R}\left(t, t_{0}, \lambda^{\infty}\right)\right)}=0
$$

where we use notation (2.13) with $\Omega=B_{R}$.

We will prove the above theorem in several steps. Let $\varepsilon>0$. We begin by considering the graphs containing big jumps of either $u$ or $v$. In view of (4.1) we have $\left\{\mathcal{S}_{u\left(t_{0}\right), \varepsilon, R}^{\beta}\right\}=\left\{\mathcal{S}_{v\left(t_{0}\right), \varepsilon, R}^{\beta}\right\}$. Let us fix a graph $\mathcal{S} \in\left\{\mathcal{S}_{u\left(t_{0}\right), \varepsilon, R}^{\beta}\right\}$ and let $\gamma: B_{R}^{d-1} \rightarrow \mathbb{R}$ be the corresponding parametrization, $\tilde{\gamma}(\bar{y})=(\bar{y}, \gamma(\bar{y})), \bar{y} \in B_{R}^{d-1}$, where $B_{R}^{d-1}$ denotes the open ball in $\mathbb{R}^{d-1}$ of radius $R$ centered at the origin. Let $\mathcal{S}_{u}$ and $\mathcal{S}_{v}$ be the graphs in $\mathbb{R}^{d+1}$ corresponding to $u$ and $v$, respectively, whose section at $B_{R} \times\left\{t=t_{0}\right\}$ coincide with $\mathcal{S}$. Finally, let $D_{t_{0}, R} \subset B_{R}^{d-1}$ be given by

$$
D_{t_{0}, R} \times\left\{t=t_{0}\right\}=D_{u} \cap\left(B_{R}^{d-1} \times\left\{t=t_{0}\right\}\right)=D_{v} \cap\left(B_{R}^{d-1} \times\left\{t=t_{0}\right\}\right),
$$

where $D_{u}$ and $D_{v}$ are the sets described in (A3) corresponding to $\mathcal{S}_{u}$ and $\mathcal{S}_{v}$, respectively. Note that $\mathcal{H}^{d-1}\left(B_{R}^{d-1}-D_{R, t_{0}}\right)=0$. Here and in what follows we include in $D_{u}$ and $D_{v}$ the points of continuity of $u$ and $v$ according with Remark 2.7 and, thus, we simply refer to assumption (A3). We also set

$$
N_{\varepsilon}=\#\left\{\mathcal{S}_{u\left(t_{0}\right), \varepsilon, R}^{\beta}\right\}, \quad L_{\varepsilon}=\mathcal{H}^{d-1}\left(S_{u\left(t_{0}\right), \varepsilon, R}\right) .
$$


In view of Assumption (A3) and by applying Besicovitch's covering theorem (see, e.g., [16], p. 35) we may select a countable family of disjoint closed balls, $\left\{B\left(\bar{y}_{i} ; \delta_{i}\right)\right\}_{i=1}^{\infty}$ so that the following four properties hold:

(1) for $\mathcal{H}^{d-1}$-almost every $\bar{y} \in B\left(\bar{y}_{i} ; \delta_{i}\right)$ and $\left(\bar{y}_{i}, \gamma\left(\bar{y}_{i}\right)\right) \in \mathcal{J}_{u\left(t_{0}\right)} \cap$ $\mathcal{J}_{u}^{t_{0}} \cap \mathcal{J}_{v}^{t_{0}}$, one has $\left|\left[u\left(\bar{y}, t_{0}\right)\right]\right|>0$

(2) if $\bar{y}_{i}$ is such that $u\left(t_{0}\right)_{+}\left(\bar{y}_{i}, \gamma\left(\bar{y}_{i}\right)\right)=u\left(t_{0}\right)_{-}\left(\bar{y}_{i}, \gamma\left(\bar{y}_{i}\right)\right)$ then

$$
\operatorname{osc}\left(u\left(t_{0}\right) \mid B\left(\tilde{\gamma}\left(\bar{y}_{i}\right) ; 2 \delta_{i}\right)\right)<\frac{\varepsilon^{2}}{N_{\varepsilon}^{2} L_{\varepsilon}} ;
$$

(3) moreover

$$
\begin{aligned}
& \limsup _{h \rightarrow 0} \frac{1}{2 \lambda^{\infty} h^{2}} \int_{t_{0}}^{t_{0}+h} d t \int_{-\lambda^{\infty} h}^{\lambda^{\infty} h} d s \int_{\substack{y_{d}-\gamma(\bar{y})=s \\
\left|\bar{y}-\bar{y}_{i}\right|<\delta_{i}}}(|u(y, t)-\bar{u}(y, t)|+|v(y, t)-\bar{v}(y, t)|) d \mathcal{H}^{d-1}(y) \\
& <\frac{\varepsilon^{2} \mathcal{H}^{d-1}\left(\mathcal{S}_{\bar{y}_{i}, \delta_{i}}\right)}{3 N_{\varepsilon}^{2} L_{\varepsilon}}
\end{aligned}
$$

where $\mathcal{S}_{\bar{y}_{i}, \delta_{i}}=\tilde{\gamma}\left(B\left(\bar{y}_{i} ; r_{i}\right)\right), \bar{u}$ and $\bar{v}$ are defined as in Assumption (A3);

(4) and

$$
\mathcal{H}^{d-1}\left(\tilde{\gamma}\left(D_{t_{0}, R}-\bigcup_{i} B\left(\bar{y}_{i} ; r_{i}\right)\right)\right)=0 .
$$

On the other hand, by Rankine-Hugoniot's relation, for all $j \in$ $\{1, \ldots, n\}$

$$
\frac{\left[\sum_{k=1}^{d} \nu_{k}\left(x, t_{0}\right) f_{j}^{k}\left(u\left(x, t_{0}\right)\right)\right]}{\left[u_{j}\left(x, t_{0}\right)\right]}=\frac{\left[\sum_{k=1}^{d} \nu_{k}\left(x, t_{0}\right) f_{j}^{k}\left(v\left(x, t_{0}\right)\right)\right]}{\left[v_{j}\left(x, t_{0}\right)\right]},
$$

whenever $\left(x, t_{0}\right) \in \tilde{\gamma}\left(D_{t_{0}, R}\right)$ is such that $\left|\left[u\left(x, t_{0}\right)\right]\right|=\left|\left[v\left(x, t_{0}\right)\right]\right|>0$. So, for such $\left(x, t_{0}\right)$, we have

$$
T_{\left(x, t_{0}\right)}\left(\mathcal{S}_{u}\right)=T_{\left(x, t_{0}\right)}\left(\mathcal{S}_{v}\right) .
$$

Hence, by the $C^{1}$-regularity of $\mathcal{S}_{u}$ and $\mathcal{S}_{v}$, if $\left(\bar{y}_{i}, \gamma\left(\bar{y}_{i}\right)\right) \in \mathcal{J}_{u\left(t_{0}\right)} \cap \mathcal{J}_{u}^{t_{0}} \cap$ $\mathcal{J}_{v}^{t_{0}}$, we have

$$
\lim _{h \rightarrow 0} \frac{1}{2 \lambda^{\infty} h^{2}} \int_{t_{0}}^{t_{0}+h} d t \int_{-\lambda^{\infty} h}^{\lambda^{\infty} h} d s \int_{\begin{array}{c}
y_{d}-\gamma(\bar{y})=s \\
\left|\bar{y}-\bar{y}_{i}\right|<\delta_{i}
\end{array}}|\bar{u}(y, t)-\bar{v}(y, t)| d \mathcal{H}^{d-1}(y)=0 .
$$


Furthermore, if $u\left(t_{0}\right)^{+}\left(\bar{y}_{i}, \gamma\left(\bar{y}_{i}\right)\right)=u\left(t_{0}\right)^{-}\left(\bar{y}_{i}, \gamma\left(\bar{y}_{i}\right)\right)$, then

$$
\begin{aligned}
\limsup _{h \rightarrow 0} \frac{1}{2 \lambda^{\infty} h^{2}} \int_{t_{0}}^{t_{0}+h} d t \int_{-\lambda^{\infty} h}^{\lambda^{\infty} h} d s \int_{\substack{y_{d}-\gamma(\bar{y})=s \\
\left|\bar{y}-\bar{y}_{i}\right|<\delta_{i}}} \mid \bar{u}(y, t) & -\bar{v}(y, t) \mid d \mathcal{H}^{d-1}(y) \\
& <\frac{\varepsilon^{2} \mathcal{H}^{d-1}\left(\mathcal{S}_{\bar{y}_{i}, \delta_{i}}\right)}{3 N_{\varepsilon}^{2} L_{\varepsilon}},
\end{aligned}
$$

may be obtained directly from the smallness of the oscillation for $y \in$ $B\left(\tilde{\gamma}\left(\bar{y}_{i}\right) ; 2 \delta_{i}\right)$. In this way, we can find $N_{0} \in \mathbb{N}$ and $h_{0}>0$ such that

$$
\mathcal{H}^{d-1}\left(\tilde{\gamma}\left(D_{t_{0}, R}-\bigcup_{i=1}^{N_{0}} B\left(\bar{y}_{i} ; r_{i}\right)\right)\right)<\frac{\varepsilon^{2}}{6 K N_{\varepsilon}^{2} L_{\varepsilon}},
$$

where $K \geq \max \left\{\|u\|_{\infty},\|v\|_{\infty}\right\}$, and

$$
\begin{gathered}
\frac{1}{2 \lambda^{\infty} h^{2}} \int_{t_{0}}^{t_{0}+h} d t \int_{-\lambda \infty h}^{\lambda^{\infty} h} d s \int_{\begin{array}{c}
y_{d}-\gamma(\bar{y})=s \\
\left|\bar{y}-\bar{y}_{i}\right|<\delta_{i}
\end{array}}|u(y, t)-v(y, t)| d \mathcal{H}^{d-1}(y)<\frac{2 \varepsilon^{2} \mathcal{H}^{d-1}\left(\mathcal{S}_{\bar{y}_{i}, \delta_{i}}\right)}{3 N_{\varepsilon}^{2} L_{\varepsilon}} \\
\quad \text { for } 0<h<h_{0} \text { and } i=1, \ldots, N_{0}
\end{gathered}
$$

Therefore, in the neighborhood of large shocks, the $L^{1}$ distance between the solutions $u$ and $v$ is estimated as

$$
\frac{1}{2 \lambda^{\infty} h^{2}} \int_{t_{0}}^{t_{0}+h} d t \int_{-\lambda^{\infty} h}^{\lambda^{\infty} h} d s \int_{\substack{y_{d}-\gamma(\bar{y})=s \\|\bar{y}|<R}}|u(y, t)-v(y, t)| d \mathcal{H}^{d-1}(y)<\frac{\varepsilon^{2}}{N_{\varepsilon}^{2}},
$$

Finally, we apply the following simple lemma from [2] (see also [24], Chap. 10).

Lemma 4.1. Let $w:(a, b) \times[0, h] \rightarrow \mathbb{R}$ be a bounded and measurable function satisfying the $L^{1}$ Lipschitz continuity property

$$
\left\|w\left(\tau_{2}\right)-w\left(\tau_{1}\right)\right\|_{L^{1}(a, b)} \leq K\left|\tau_{2}-\tau_{1}\right|, \quad \tau_{1}, \tau_{2} \in[0, h],
$$

for some constant $K>0$. Then, we have

$$
\frac{1}{h} \int_{a}^{b}|w(\xi, h)| d \xi \leq \sqrt{2 K}\left(\frac{1}{h^{2}} \int_{0}^{h} \int_{a}^{b}|w| d \xi d \tau\right)^{1 / 2}
$$

whenever the right-hand side is less than $K$. 
Set

and

$$
\gamma^{\beta}=\gamma_{u\left(t_{0}\right)}^{\beta}=\gamma_{v\left(t_{0}\right)}^{\beta}
$$

$$
W^{\beta}(h)=\left\{y \in B_{R}: \gamma^{\beta}(\bar{y})-\lambda^{\infty} h \leq y_{d} \leq \gamma^{\beta}(\bar{y})+\lambda^{\infty} h\right\} .
$$

We have

$$
\begin{aligned}
& \frac{1}{h} \int_{\cup_{\beta} W^{\beta}(h)}\left|u\left(x, t_{0}+h\right)-v\left(x, t_{0}+h\right)\right| d x \\
& \leq \sum_{\beta=1}^{N_{\varepsilon}} \frac{1}{h} \int_{-\lambda^{\infty} h}^{\lambda^{\infty} h}\left(\int_{\substack{y_{d}-\gamma^{\beta}(\bar{y})=s \\
|\bar{y}|<R}}\left|u\left(y, t_{0}+h\right)-v\left(y, t_{0}+h\right)\right| d \mathcal{H}^{d-1}(y)\right) d s \\
& \leq 2 \sqrt{\lambda^{\infty} K} \sum_{\beta=1}^{N_{\varepsilon}}\left(\frac{1}{2 \lambda^{\infty} h^{2}} \int_{t_{0}}^{t_{0}+h} \int_{-\lambda^{\infty} h}^{\lambda^{\infty} h}\left(\underset{\substack{y_{d}-\gamma^{\beta}(\bar{y})=s \\
|\bar{y}|<R}}{\int}|u(y, t)-v(y, t)| d \mathcal{H}^{d-1}(y)\right) d s d t\right)^{1 / 2}
\end{aligned}
$$

We have thus arrived at the following estimate

$$
\frac{1}{h} \int_{\cup_{\beta} W^{\beta}(h)}\left|u\left(x, t_{0}+h\right)-v\left(x, t_{0}+h\right)\right| d x<2 \sqrt{\lambda^{\infty} K} \varepsilon .
$$

We now deal with the regions where the pointwise oscillation is less than or equal to $\varepsilon$. We have

$$
B_{R} \backslash S_{u\left(t_{0}\right), \varepsilon, R}=\cup_{j=1}^{N_{\varepsilon}^{\prime}} \Omega_{\varepsilon}^{j},
$$

and, for each $x \in \bar{\Omega}_{\varepsilon}^{j}, j=1, \ldots, N_{\varepsilon}^{\prime}$,

$$
\operatorname{osc}_{x} u\left(t_{0}\right)=\operatorname{osc}_{x} v\left(t_{0}\right) \leq \varepsilon \text {. }
$$

We fix $j=j_{0}$ and set $\Omega=\Omega_{\varepsilon}^{j_{0}}$. We take a covering of $\bar{\Omega}$ by a finite number of ball,

$$
\bar{\Omega} \subset \bigcup_{l=1}^{M_{\varepsilon}} B\left(x_{l} ; r_{l}\right),
$$

with

$$
\operatorname{osc}\left(u\left(t_{0}\right) \mid B\left(x_{l} ; r_{l}\right)\right) \leq 2 \varepsilon, \quad l=1, \ldots, M_{\varepsilon},
$$

such that each one of the balls $B\left(x_{l} ; r_{l}\right)$ intersects at most $N_{d}$ such other balls, where $N_{d}$ depends only on the dimension $d$. We may find $h_{1}=h_{1 j_{0}}>0$ such that

$$
\Omega\left(t, t_{0}, \lambda^{\infty}\right) \subset \bigcup_{l=1}^{M_{\varepsilon}} D\left(B\left(x_{l} ; r_{l}\right), t_{0}, \lambda^{\infty}\right), \quad t_{0} \leq t \leq t_{0}+h_{1} .
$$


We set, for short, $B_{l}=B\left(x_{l} ; r_{l}\right)$. Then

$$
\bar{\Omega}\left(t_{0}+h, t_{0}, \lambda^{\infty}\right) \subset \bigcup_{l=1}^{M_{\varepsilon}} B_{l}\left(t_{0}+h, t_{0}, \lambda^{\infty}\right), \quad \text { for } 0<h \leq h_{1} .
$$

Let also

$$
\Omega_{l}\left(t_{0}+h, t_{0}\right):=\Omega\left(t_{0}+h, t_{0}, \lambda^{\infty}\right) \cap B_{l}\left(t_{0}+h, t_{0}, \lambda^{\infty}\right) .
$$

Define $\tilde{u}$ out of a set of $H^{d}$-measure zero by

$$
\tilde{u}(x, t):=\frac{u_{+}+u_{-}}{2} .
$$

Fix $l \in\left\{1, \ldots, M_{\varepsilon}\right\}$. Set

$$
\underline{A}^{k}:=A^{k}\left(\tilde{u}\left(x_{l}, t_{0}\right)\right), \quad \underline{\lambda}_{j}^{k}:=\lambda_{j}^{k}\left(\tilde{u}\left(x_{l}, t_{0}\right)\right), \quad \underline{l}_{j}:=l_{j}\left(\tilde{u}\left(x_{l}, t_{0}\right)\right) .
$$

Let $\underline{u}(x, t)$ be the solution of the linear hyperbolic problem

$$
\begin{aligned}
& \partial_{t} \underline{u}+\sum_{k=1}^{d} \underline{A}^{k} \partial_{k} \underline{u}=0, \quad t \geq t_{0}, \\
& \underline{u}\left(t_{0}\right)=u\left(t_{0}\right) .
\end{aligned}
$$

For $(x, t) \in \mathcal{J}_{u}$, let $\psi\left(u_{-}, u_{+}\right)$be the shock speed given by RankineHugoniot relation, that is,

$$
\psi\left(u_{-}, u_{+}\right)[u]=\sum_{k=1}^{d} \nu_{k}\left[f^{k}(u)\right]
$$

where $\nu=\left(\nu_{1}, \ldots, \nu_{d}\right)$ is the unit normal to $\mathcal{J}_{u(t)}$ at $x$. We may write (1.1) in the form

$$
\partial_{t} u+\sum_{k=1}^{d} A^{k}(\tilde{u}) \partial_{k} u=\mu
$$

where

$$
\mu=\left(\sum_{k=1}^{d} \nu_{k} A^{k}(\tilde{u})-\psi\left(u_{-}, u_{+}\right)\right)\left(u_{+}-u_{-}\right) \mathcal{H}^{d}\left\lfloor\mathcal{J}_{u}\right.
$$

We rewrite (4.10) as

$$
\partial_{t} u+\sum_{k=1}^{d} \underline{A}^{k} \partial_{k} u=\mu+\sum_{k=1}^{d}\left(\underline{A}^{k}-A^{k}(\tilde{u})\right) \partial_{k} u .
$$


Taking the difference of (4.12) and (4.8) and multiplying by $\underline{l}_{j}, j=$ $1, \ldots, N$, we arrive at

$$
\partial_{t}\left(\underline{l}_{j}(u-\underline{u})\right)+\sum_{k=1}^{d} \underline{\lambda}_{j}^{k} \partial_{k}\left(\underline{l}_{j}(u-\underline{u})\right)=\underline{l}_{j} \mu+\sum_{k=1}^{d} \underline{l}_{j}\left(\underline{A}^{k}-A^{k}(\tilde{u})\right) \partial_{k} u .
$$

For $x_{0} \in \Omega_{l}\left(t_{0}+h, t_{0}\right)$ and $r>0$ such that $B\left(x_{0} ; r\right) \in \Omega_{l}\left(t_{0}+h, t_{0}\right)$, let

$$
\begin{gathered}
C^{j}\left(x_{0} ; r\right)=C^{j}\left[B\left(x_{0} ; r\right)\right]:=\left\{(x, t) \in \mathbb{R}^{d} \times(0, T):\right. \\
\left.\left(x_{1}+\underline{\lambda}_{j}^{1}\left(t_{0}+h-t\right), \ldots, x_{d}+\underline{\lambda}_{j}^{d}\left(t_{0}+h-t\right)\right) \in B\left(x_{0} ; r\right), t_{0} \leq t \leq t_{0}+h\right\}
\end{gathered}
$$

By Gauss-Green's formula we have

$$
\begin{aligned}
\int_{C^{j}\left(x_{0} ; r\right)}\left\{\partial_{t}\left(\underline{l}_{j}(u-\underline{u})\right)+\sum_{k=1}^{d}\right. & \left.\underline{\lambda}_{j}^{k} \partial_{k}\left(\underline{l}_{j}(u-\underline{u})\right)\right\} d x d t \\
& =\int_{B\left(x_{0} ; r\right)} \underline{l}_{j}(u-\underline{u})\left(x, t_{0}+h\right) d x .
\end{aligned}
$$

On the other hand, by the controled oscillation property we have both

$$
\left|\underline{l}_{j} \mu\left(C^{j}\left(x_{0} ; r\right)\right)\right| \leq O(\varepsilon) \int_{t_{0}}^{t_{0}+h} \mathrm{TV}\left(u(t) \mid C_{t}^{j}\left(x_{0} ; r\right)\right) d t
$$

and

$$
\left|\underline{l}_{j}\left(\underline{A}^{k}-A^{k}(\tilde{u})\right) \partial_{k} u\left(C^{j}\left(x_{0} ; r\right)\right)\right| \leq C \varepsilon \int_{t_{0}}^{t_{0}+h} \mathrm{TV}\left(u(t) \mid C_{t}^{j}\left(x_{0} ; r\right)\right) d t
$$

where $C_{s}^{j}\left(x_{0} ; r\right)=C^{j}\left(x_{0} ; r\right) \cap\{t=s\}$. Combining (4.14), (4.15) and (4.16), we arrive at the estimate

$$
\left|\int_{B\left(x_{0} ; r\right)} \underline{l}_{j}(u-\underline{u})\left(x, t_{0}+h\right) d x\right| \leq O(\varepsilon) \int_{t_{0}}^{t_{0}+h} \mathrm{TV}\left(u(t) \mid C_{t}^{j}\left(x_{0} ; r\right)\right) d t .
$$

Now we have the following simple lemma.

Lemma 4.2. Let $\mu_{1}, \mu_{2}$ be two Radon measures defined on the open set $\Omega \subset \mathbb{R}^{d}$, with $\mu_{2} \geq 0$. Assume that for all $x_{0} \in \Omega$ and $r>0$, such that $B\left(x_{0} ; r\right) \subset \Omega$ we have

$$
\left|\mu_{1}\left(B\left(x_{0} ; r\right)\right)\right| \leq \mu_{2}\left(B\left(x_{0} ; r\right)\right) .
$$

Then, we have

$$
\left|\mu_{1}\right| \leq \mu_{2}
$$


Proof. First we note that the inequality (4.18) extends to all open sets $\Omega^{\prime} \subset \Omega$. Indeed, by Besicovitch's covering theorem, we may find a countable disjoint family of open balls $\left\{B_{\alpha}\right\}$, with $\bigcup_{\alpha} B_{\alpha} \subset \Omega^{\prime}$ and

$$
\left(\left|\mu_{1}\right|+\mu_{2}\right)\left(\Omega^{\prime} \backslash \bigcup_{\alpha} B_{\alpha}\right)=0
$$

Hence,

$$
\left|\mu_{1}\left(\Omega^{\prime}\right)\right|=\left|\mu_{1}\left(\bigcup_{\alpha} B_{\alpha}\right)\right| \leq \sum_{\alpha}\left|\mu_{1}\left(B_{\alpha}\right)\right| \leq \sum_{\alpha} \mu_{2}\left(B_{\alpha}\right)=\mu_{2}\left(\bigcup_{\alpha} B_{\alpha}\right)=\mu_{2}\left(\Omega^{\prime}\right) .
$$

It also extends to compact sets. Indeed, given a compact $K \subset \Omega$, denoting $K_{\delta}=\{x \in \Omega: \operatorname{dist}(x, K)<\delta\}$, we have

$$
\left|\mu_{1}(K)\right|=\lim _{\delta \rightarrow 0}\left|\mu_{1}\left(K_{\delta}\right)\right| \leq \lim _{\delta \rightarrow 0} \mu_{2}\left(K_{\delta}\right)=\mu_{2}(K) .
$$

Finally, let $\Omega^{-}$and $\Omega^{+}$be two disjoint Borel sets such that $\Omega=\Omega^{+} \cup \Omega^{-}$ and $\mu_{1} \geq 0$ over $\Omega^{+}, \mu_{1} \leq 0$, over $\Omega^{-}$. Given any Borel set $B \subset \Omega$, set $B^{+}=B \cap \Omega^{+}, B^{-}=B \cap \Omega^{-}$. We have

$$
\begin{aligned}
& \left|\mu_{1}\left(B^{ \pm}\right)\right|=\sup \left\{\left|\mu_{1}\right|(K): K \subset B^{ \pm}, \text {compact }\right\} \\
& \quad \leq \sup \left\{\mu_{2}(K): K \subset B^{ \pm}, \text {compact }\right\}=\mu_{2}\left(B^{ \pm}\right),
\end{aligned}
$$

and so, we arrive at

$$
\left|\mu_{1}\right|(B)=\left|\mu_{1}\left(B^{+}\right)\right|+\left|\mu_{1}\left(B^{-}\right)\right| \leq \mu_{2}\left(B^{+}\right)+\mu_{2}\left(B^{-}\right)=\mu_{2}(B) .
$$

Applying Lemma 4.2 to (4.17), we obtain

$$
\int_{\Omega_{l}\left(t_{0}+h, t_{0}\right)}\left|\underline{l}_{j}(u-\underline{u})\left(x, t_{0}+h\right)\right| d x \leq O(\varepsilon) \int_{t_{0}}^{t_{0}+h} \mathrm{TV}\left(u(t) \mid \Omega_{l}\left(t, t_{0}\right)\right) d t .
$$

Analogously, we have

$$
\int_{\Omega_{l}\left(t_{0}+h, t_{0}\right)}\left|\underline{l}_{j}(v-\underline{u})\left(x, t_{0}+h\right)\right| d x \leq O(\varepsilon) \int_{t_{0}}^{t_{0}+h} \mathrm{TV}\left(v(t) \mid \Omega_{l}\left(t, t_{0}\right)\right) d t
$$

and, so,

$$
\begin{aligned}
& \int\left|(u-v)\left(x, t_{0}+h\right)\right| d x \\
\Omega_{l}\left(t_{0}+h, t_{0}\right) & \\
\leq & O(\varepsilon) \int_{t_{0}}^{t_{0}+h}\left(\operatorname{TV}\left(u(t) \mid \Omega_{l}\left(t, t_{0}\right)\right)+\operatorname{TV}\left(v(t) \mid \Omega_{l}\left(t, t_{0}\right)\right)\right) d t .
\end{aligned}
$$


Since each one of the balls $B\left(x_{l} ; r_{l}\right)$ intersects at most $N_{d}$ such other balls, where $N_{d}$ depends only on the dimension $d$, we further conclude

$$
\begin{aligned}
& \quad \int\left|(u-v)\left(x, t_{0}+h\right)\right| d x \\
& \leq O(\varepsilon) \int_{t^{j}\left(t_{0}+h, t_{0}\right)}^{t_{0}+h}\left(\mathrm{TV}\left(u(t) \mid \cup \Omega^{j}\left(t, t_{0}\right)\right)+\mathrm{TV}\left(v(t) \mid \cup \Omega^{j}\left(t, t_{0}\right)\right)\right) d t \\
& \leq h O(\varepsilon) \sup _{t_{0} \leq t \leq t_{0}+h}\left(\mathrm{TV}\left(u(t) \mid B_{R}\left(t, t_{0}\right)\right)+\mathrm{TV}\left(v(t) \mid B_{R}\left(t, t_{0}\right)\right)\right) .
\end{aligned}
$$

Hence, setting $h_{*}=\min \left\{h_{0}, h_{1 j}, j=1, \ldots, N_{\varepsilon}^{\prime}\right\}$, from (4.7) and (4.21), for $0<h \leq h_{*}$, we have

$$
\begin{aligned}
& \frac{1}{h} \int_{B_{R}\left(t_{0}+h, t_{0}\right)}\left|u\left(x, t_{0}+h\right)-v\left(x, t_{0}+h\right)\right| d x \\
\leq & 2 \sqrt{\lambda^{\infty} K} \varepsilon+O(\varepsilon) \sup _{t_{0} \leq t \leq t_{0}+h}\left(\operatorname{TV}\left(u(t) \mid B_{R}\left(t, t_{0}\right)\right)+\mathrm{TV}\left(v(t) \mid B_{R}\left(t, t_{0}\right)\right)\right) .
\end{aligned}
$$

Since $\varepsilon>0$ is arbitrary, this concludes the proof of Theorem 4.1.

Conclusion of the proof of Theorem 3.1. We conclude the proof of Theorem 3.1 by applying the following lemma whose proof maybe found in $[6,24]$.

Lemma 4.3. For every $u_{0} \in \mathcal{K}$ and every Lipschitz continuous map $u:[0, T] \rightarrow L^{1}\left(\mathbb{R}^{d}\right)$, with $u(t) \in \mathcal{K}$, for all $t \in[0, T]$, and $u(0)=u_{0}$, the semi-group of solutions $S: \mathcal{K} \times[0, \infty) \rightarrow \mathcal{K}$ satisfies the estimate

$$
\begin{aligned}
& \int_{\Omega\left(t_{1}, 0\right)}\left|u\left(x, t_{1}\right)-\left(S\left(t_{1}\right) u_{0}\right)(x)\right| d x \\
& \quad \leq K \int_{0}^{t_{1}} \liminf _{h \rightarrow 0} \frac{1}{h}\left(\int_{\Omega(t+h, 0)}|u(x, t+h)-(S(h) u(t))(x)| d x\right) d t
\end{aligned}
$$

for any open set $\Omega \subset \mathbb{R}^{d}$ and all $t_{1} \in(0, T)$, where we use notation (2.13) ommiting $\lambda=\lambda^{\infty}$.

Observing that $\Omega(t+h, 0)=\Omega_{t}(t+h, t)$, where $\Omega_{t}=\Omega(t, 0)$, we obtain from Theorem 4.1 that the integrand in the right-hand side of (4.23) vanishes for almost all $t \in[0, T]$. The application of Theorem 4.1 is justified once we show that, for any $t_{0}>0$, if $t_{0}$ is not an interaction time for $u$ then $t_{0}$ is not an interaction time for $u_{t_{0}}^{*}(x, t):=S\left(t-t_{0}\right) u\left(t_{0}\right)$, $t \geq t_{0}$, which, by property (5) of semigroups, will be established if we 
show that: (i) $\left(x_{0}, t_{0}\right) \in \mathcal{C}_{u\left(t_{0}\right)}$ implies $\left(x_{0}, t_{0}\right) \in \mathcal{C}_{u_{t_{0}}^{*}}$; (ii) if $\left(x_{0}, t_{0}\right)$ is a density point of $\mathcal{J}_{u\left(t_{0}\right)} \cap \mathcal{J}_{u}^{t_{0}}$, where a unit normal to $\mathcal{J}_{u\left(t_{0}\right)}, \nu$, is defined, then $\left(x, t_{0}\right) \in \mathcal{J}_{u_{t_{0}}^{*}}$. The locally controlled oscillation property immediately gives (i). Assertion (ii), on the other hand, follows from properties (2), (4) and(6) of continuous semigroups. Indeed, we have

$$
\lim _{\rho \rightarrow 0} \frac{1}{\left|B\left(x_{0} ; \rho\right)\right|} \int_{B\left(x_{0} ; \rho\right)}\left|u\left(t_{0}\right)(x)-\bar{u}_{0}\left(x-x_{0}\right)\right| d x=0,
$$

where $\bar{u}_{0}$ is as in property (4) of Definition 3.2. Hence, using properties (2), (4) and (6) of Definition 3.2, we get

$$
\lim _{\rho \rightarrow 0} \frac{1}{\rho\left|B\left(x_{0} ; \rho\right)\right|} \int_{t_{0}}^{t_{0}+\rho} \int_{B\left(x_{0} ; \rho\right)}\left|u_{t_{0}}^{*}(x, t)-\bar{u}\left(x-x_{0}, t-t_{0}\right)\right| d x d t=0,
$$

which proves the assertion. Here, again, $\bar{u}$ is as in (4) of Definition 3.2.

Hence, we conclude

$$
\int_{\Omega(t, 0)}\left|u\left(x, t_{1}\right)-\left[S\left(t_{1}\right) u_{0}\right](x)\right| d x=0, \quad \text { for all } t_{1} \in(0, T) .
$$

This completes the proof of Theorem 3.1.

\section{EXTENSION TO NONCONSERVATIVE SYSTEMS AND NONCLASSICAL ENTROPY SOLUTIONS}

In this section we extend Theorem 3.1 to systems in nonconservative form. Consider the nonlinear hyperbolic system (1.2). Following $[2,24]$, to define the notion of entropy solutions we prescribe a family of admissible discontinuities $\Phi \subset \mathcal{U} \times \mathcal{U}$ and a family of admissible speeds $\psi: \Phi \rightarrow\left(-\lambda^{\infty}, \lambda^{\infty}\right)$ satisfying the following consistency property for all pairs $\left(u_{-}, u_{+}\right) \in \Phi$ :

$$
\left|\left(\sum_{k=1}^{d} A^{k}\left(u_{+}\right) \nu_{k}-\psi\left(u_{-}, u_{+}\right)\right)\left(u_{+}-u_{-}\right)\right| \leq C\left|u_{+}-u_{-}\right|^{2},
$$

where $\nu_{k}$ is the normal to the discontinuity surface and $C>0$ is a fixed constant. We denote by $u_{+}$a pointwise representative of a $B V$ function $u$.

Definition 5.1 (General concept of entropy solution). Let $\Phi \subset \mathcal{U} \times \mathcal{U}$ be a set of admissible jumps and $\psi: \Phi \rightarrow\left(-\lambda^{\infty}, \lambda^{\infty}\right)$ be a family of admissible speeds satisfying (5.1). A function $u: \mathbb{R}^{d} \times(0, T) \rightarrow \mathcal{U}$ satisfying (A1) and (A2) in Section 2 is called a $(\Phi, \psi)$-admissible entropy solution of (1.2) or, in short, an entropy solution if the following two conditions hold: 
- The restriction of the measure $\partial_{t} u+\sum_{k=1}^{d} A^{k}\left(u_{+}\right) \partial_{k} u$ to the set $\mathcal{C}_{u}$ vanishes identically, that is,

$$
\iint_{B} \partial_{t} u+\sum_{k=1}^{d} A^{k}\left(u_{+}\right) \partial_{k} u=0 \quad \text { for every Borel set } B \subset \mathcal{C}_{u} .
$$

- at each point $(x, t) \in \mathcal{J}_{u}$, such that $x \in \mathcal{J}_{u(t)}$ and the upward (with respect to the corresponding graphs) unit normals $\tilde{\nu}(x, t)$ and $\nu(x, t)$, to $\mathcal{J}_{u}$ and $\mathcal{J}_{u(t)}$, respectively, are defined, the limits $u_{ \pm}(x, t)$ and the speed $\lambda^{u}(x, t)$, determined by

$$
\tilde{\nu}(x, t)=\frac{1}{\left(1+\lambda^{u}(x, t)^{2}\right)^{1 / 2}}\left(\nu(x, t),-\lambda^{u}(x, t)\right),
$$

satisfy

$$
\left(u_{-}(x, t), u_{+}(x, t)\right) \in \Phi, \quad \lambda^{u}(x, t)=\psi\left(u_{-}(x, t), u_{+}(x, t)\right) .
$$

From (5.1), (5.2), and (5.3) we deduce that if $u$ is an entropy solution then, for every Borel set $B$,

$$
\begin{aligned}
& \iint_{B}\left(\partial_{t} u+\sum_{k=1}^{d} A^{k}\left(u_{+}\right) \partial_{k} u\right) \\
& =\iint_{B \cap \mathcal{C}(u)}\left(\partial_{t} u+\sum_{k=1}^{d} A^{k}\left(u_{+}\right) \partial_{k} u\right)+\int_{B \cap \mathcal{J}(u)}\left(-\lambda^{u}+\sum_{k=1}^{d} A^{k}\left(u_{+}\right) \nu_{k}\right)\left(u_{+}-u_{-}\right) d \mathcal{H}^{d} \\
& =\int_{B \cap \mathcal{J}(u)}\left(\sum_{k=1}^{d} A^{k}\left(u_{+}\right) \nu_{k}-\psi\left(u_{-}, u_{+}\right)\right)\left(u_{+}-u_{-}\right) d \mathcal{H}^{d} .
\end{aligned}
$$

A continuous semigroup of $(\Phi, \psi)$-admissible entropy solutions for (1.2) is defined in exactly the same way as in Section 3. Then, by the same arguments as the ones used for Theorem 3.1, we arrive at the following uniqueness result for hyperbolic systems (1.2) which extend to the multidimensional context the result in [2] for the one-dimensional case.

Theorem 5.1. (Uniqueness of $(\Phi, \psi)$-admissible solutions.) Let $\Phi \subset \mathcal{U} \times \mathcal{U}$ be a set of admissible jumps and $\psi: \Phi \rightarrow\left(-\lambda^{\infty}, \lambda^{\infty}\right)$ be a family of admissible speeds satisfying (5.1). Suppose that there exists a continuous semigroup of $(\Phi, \psi)$-admissible entropy solutions of (1.2), defined on some non-empty subset $\mathcal{K}$ of $B V\left(\mathbb{R}^{d} ; \mathcal{U}\right)$, satisfying the following consistency property with single jumps: If a function $v=v(x, t)$ is made of a single (admissible) jump discontinuity $\left(v_{-}, v_{+}\right) \in \Phi$ propagating with the speed $\psi\left(v_{-}, v_{+}\right)$, then $v(0) \in \mathcal{K}$ and

$$
v(t)=S(t) v(0), \quad t \geq 0 .
$$


Then, given any $u_{0} \in \mathcal{K}$ and $T>0, u^{*}(x, t)=\left(S(t) u_{0}\right)(x)$ is the only entropy solution $u$ of $(1.2),(3.3)$ satisfying $u \in \mathcal{O}\left(0, T ; B V^{\text {roc }}\left(\mathbb{R}^{d}\right)\right)$ and $u(t) \in \mathcal{K}$, for all $t \in[0, T]$.

It is clear that the consistency property above is necessary for uniqueness, for otherwise one could find two distinct solutions starting with the same initial data and the conclusion of Theorem 5.1 would obviously fail.

\section{ACKNOWLEDGEMENTS}

The first author gratefully acknowledges the support received from CNPq through the grants 352871/96-2 and FAPERJ through the grant E-26/152.192/2002. This work was done when the second author was visiting the Instituto de Matemática Pura e Aplicada, Rio de Janeiro, in December 2003, thanks to the CNRS-CNPq France-Brazil Agreement.

\section{REFERENCES}

[1] L. Ambrosio, F. Bouchut, and C. De Lellis, Well-posedness for a class of hyperbolic systems of conservation laws in several space dimensions, Comm. Partial Diff. Equa. 29 (2004), 1635-1651.

[2] P. Baiti, P.G. LeFloch, and B. Piccoli, Uniqueness of classical and nonclassical solutions for nonlinear hyperbolic systems, J. Differential Equations 172 (2001), 59-82.

[3] S. Bianchini, Interaction estimates and Glimm functionals for general hyperbolic systems, Discrete Contin. Dyn. Syst. 9 (2003),133-166.

[4] S. Bianchini and A. Bressan, Vanishing viscosity solutions of nonlinear hyperbolic systems, Annals Math. (2005), to appear.

[5] P. Brenner, The Cauchy problem for symmetric hyperbolic systems in $L^{p}$, Math. Scand. 19 (1966), 27-37.

[6] A. Bressan, Hyperbolic Systems of Conservation Laws. The one-dimensional Cauchy problem, Oxford Univ. Press, 2000.

[7] A. Bressan and P. Goatin, Oleinik-type estimates and uniqueness for $n \times n$ conservation laws, J. Differential Equations 156 (1999), 26-49.

[8] A. Bressan and P.G. LeFloch, Uniqueness of entropy solutions for systems of conservation laws, Arch. Rational Mech. Anal. 140 (1999), 301-331.

[9] A. Bressan and M. Lewicka, A uniqueness condition for hyperbolic conservation laws, Discrete Contin. Dynam. Systems 6 (2000), 673-682.

[10] C.M. Dafermos, The entropy rate admissible criterion for solutions of hyperbolic conservation laws, J. Differential Equations 14 (1973), 202-212.

[11] C.M. Dafermos, Generalized characteristics and the structure of solutions of hyperbolic conservation laws, Indiana Univ. Math. J. 26 (1977), 1097-1119.

[12] C.M. Dafermos, Stability for systems of conservation laws in several space dimensions, SIAM J. Math. Anal. 26 (1995), 1403-1414.

[13] C.M. Dafermos, Hyperbolic conservation laws in continuum physics, Grundlehren Math. Wissenschaften Series 325, Springer Verlag, 2000. 
[14] G. Dal Maso, P.G. LeFloch, and F. Murat, Definition and weak stability of nonconservative products, J. Math. Pure Appl. 74 (1995), 483-548.

[15] R.J. DiPerna, Singularities of solutions of nonlinear hyperbolic systems of conservation laws, Arch. Rational Mech. Anal. 60 (1976), 75-100.

[16] C. Evans and R.F. Gariepy, Measure theory and fine properties of functions, Studies in Advanced Mathematics, CRC Press, 1992, Boca Raton, FL.

[17] H. Federer, Geometric measure theory, Springer-Verlag, New York, 1969.

[18] J. Glimm, Solutions in the large for nonlinear hyperbolic systems of equations, Comm. Pure Appl. Math. 4 (1965), 697-715.

[19] J. Glimm and P.D. Lax, Decay of solutions to nonlinear hyperbolic conservation laws, Mem. Amer. Math. Soc. 101, 1971.

[20] J.X. Hu and P. LeFloch, $L^{1}$ Continuous dependence property for systems of conservation laws, Arch. Rational Mech. Anal. 151 (2000), 45-93.

[21] T. Iguchi and P. LeFloch, Existence theory for hyperbolic systems of conservation laws with general flux-functions, Arch. Rational Mech. Anal. 168 (2003), 165-244.

[22] P.D. Lax, The formation and decay of shock waves, Amer. Math. Monthly 79 (1972), 227-241.

[23] P.D. Lax, Hyperbolic systems of conservation laws and the mathematical theory of shock waves, Regional Conf. Series in Appl. Math. 11, SIAM, Philadelphia, 1973.

[24] P.G. LeFloch, Hyperbolic systems of conservation laws: the theory of classical and nonclassical shock waves, Lecture Notes in Mathematics, ETH Zürich, Birkhäuser, 2002.

[25] P.G. LeFloch and T.-P. Liu, Existence theory for nonconservative hyperbolic systems, Forum Math. 5 (1993), 261-280.

[26] T.-P. Liu, The Riemann problem for general $2 \times 2$ conservation laws, Trans. Amer. Math. Soc. 199 (1974), 89-112.

[27] T.-P. Liu, Admissible solutions of hyperbolic conservation laws, Mem. Amer. Math. Soc. 30, 1981.

[28] T.-P. Liu and T. Yang, Weak solutions of general systems of hyperbolic conservation laws, Commun. Math. Phys. 230 (2002), 289-327.

[29] J. Rauch, BV estimates fail for most quasilinear hyperbolic systems in dimensions greater than one, Commun. Math. Phys. 106 (1986), 481-484.

(H. Frid) Instituto de Matemática Pura e Aplicada - IMPA, Estrada Dona Castorina, 110, Rio de Janeiro, RJ 22460-320, Brazil

E-mail address: hermano@impa.br

(P.G. LeFloch) Laboratoire Jacques-Louis Lions \& CNRS UMR 7598, University of Paris 6, 75252 Paris Cedex 05, France.

E-mail address: lefloch@ann.jussieu.fr 